\title{
BMJ Open Ethnic variations in risk of preterm birth in an ethnically dense socially disadvantaged area in the UK: a retrospective cross-sectional study
}

Shuby Puthussery, ${ }^{\oplus 1}$ Leah Li, ${ }^{\odot 2}$ Pei-Ching Tseng, ${ }^{1}$ Lesley Kilby, ${ }^{3}$ Jogesh Kapadia, ${ }^{3}$ Thomas Puthusserry, ${ }^{4}$ Amardeep Thind ${ }^{5}$

To cite: Puthussery S, Li L, Tseng P-C, et al. Ethnic variations in risk of preterm birth in an ethnically dense socially disadvantaged area in the UK: a retrospective crosssectional study. BMJ Open 2019;9:e023570. doi:10.1136/ bmjopen-2018-023570

- Prepublication history and additional material for this paper are available online. To view these files, please visit the journal online (http://dx.doi org/10.1136/bmjopen-2018023570).

Received 12 April 2018 Revised 12 January 2019 Accepted 4 February 2019

Check for updates

(C) Author(s) (or their employer(s)) 2019. Re-use permitted under CC BY-NC. No commercial re-use. See rights and permissions. Published by BMJ.

For numbered affiliations see end of article.

Correspondence to

Dr Shuby Puthussery;

shuby.puthussery@beds.ac.uk

\section{ABSTRACT}

Objective To investigate ethnic variations in risk of preterm birth (PTB), including extreme preterm birth (EPTB) and moderately preterm birth (MPTB), among mothers in an ethnically dense, socially disadvantaged area, and to examine whether any variations were dependent of area deprivation and maternal biological and behavioural factors.

Design Retrospective cross-sectional study using routinely collected data.

Setting A large UK National Health Service maternity unit. Participants 46307 women who gave singleton births between April 2007 and March 2016.

Outcome measures PTB defined as $<37$ weeks of gestation and further classified into EPTB ( $<28$ weeks of gestation) and MPTB (28 to $<37$ weeks of gestation). Results Overall prevalence of PTB was higher (8.3\%) compared with the national average (7.8\%). Black Caribbean (2.2\%) and black African (2.0\%) mothers had higher absolute risk of EPTB than white British mothers (1.3\%), particularly black Caribbean mothers whose relative risk ratio (RRR) was nearly twice after adjustment for all covariates ( $R R R=1.93[1.20$ to 3.10]). Excess relative risk of EPTB among black African mothers became nonsignificant after adjustment for prenatal behavioural factors (RRR=1.41[0.99 to 2.01]). Bangladeshi mothers had the lowest absolute risk of EPTB $(0.6 \%)$, substantially lower than white British mothers (1.3\%); the difference in relative risk remained significant after adjustment for area deprivation ( $R R R=0.59[0.36$ to 0.96$])$, but became non-significant after adjustment for maternal biological factors. Changes were evident in the relative risk of EPTB and MPTB among some ethnic groups compared with the white British on adjustment for different covariates. Conclusions Higher than national rates of PTB point to the need for evidence-based antenatal and neonatal care programmes to support preterm babies and their families in ethnically dense socially disadvantaged areas. Differential impact of area deprivation and the role of modifiable behavioural factors highlight the need for targeted preventive interventions for groups at risk.

\section{INTRODUCTION}

Preterm birth (PTB)—birth of a baby at fewer than 37 completed weeks of

\section{Strengths and limitations of this study}

- This is the first study that used a large recent data set to examine ethnic variations in preterm birth (PTB) in an ethnically dense area with high levels of deprivation.

- Our cohort had substantially higher proportions $(34.8 \%)$ of mothers from ethnic minority groups compared with the maternal population in England and Wales (12.9\%) allowing greater statistical power to explore differences between ethnic groups.

- Due to the paucity of adequate information, we were unable to examine ethnic variations by country of birth.

- We were unable to examine the impact of individual-level indicators of socioeconomic status (eg, education, employment and household income) on ethnic differences in PTB as they were not available.

gestation-remains a major cause of infant mortality and morbidity worldwide. Approximately 15 million babies are born preterm every year, resulting in 1 million infant deaths globally. ${ }^{1}$ In the UK, about $7.8 \%$ of babies were born preterm nationally in $2016^{2}$ and immaturity-related conditions accounted for more than half of all neonatal deaths in $2015 .^{3}$ Infant mortality rates tend to be substantially higher among preterm babies (21.1 deaths per 1000 live births) in the UK compared with babies born at term (1.4 deaths per 1000 live births). ${ }^{4}$

PTB is linked to various maternal biological and social factors including ethnicity and socioeconomic disadvantage. ${ }^{2}$ 5-12 Studies examining associations between ethnicity and PTB have shown that women from ethnic minority groups are at higher risk of PTB compared with the white population..$^{211-13}$ In a recent analysis of nationally representative data in the UK, the highest percentage of PTB was found among the black Caribbean ethnic group with $10.4 \%$ of births 
being classed as preterm. ${ }^{2}$ The same analysis reported higher prevalence of PTB among Bangladeshi, Pakistani and Indian mothers compared with the white British. ${ }^{2}$ Another study found babies of African and Caribbean ethnicities, regardless of their mothers being born in the UK, Africa or the Caribbean, tended to have lower gestational age on average compared with white British babies with UK-born mothers. ${ }^{12}$ Socioeconomic disadvantage is another risk factor that has long been linked to adverse infant outcomes including PTB. ${ }^{5-10} 14$ Studies that have used area deprivation as a measure of socioeconomic disadvantage have shown that the risk of very PTB varied between $1.6^{9}$ and $1.9^{14}$ in more deprived neighbourhoods in the UK compared with the less deprived. Bonet $e t a l^{7}$ have reported a strong association between neighbourhood socioeconomic deprivation and the risk of extreme preterm singleton birth in two European regions.

Studies that have taken into account the effect of both ethnicity and socioeconomic factors on adverse infant outcomes have indicated marked socioeconomic gradients in observed differences between white and non-white groups. ${ }^{11}{ }^{15-18}$ One UK-based study found that the prevalence of PTB increased with increasing levels of socioeconomic deprivation, and ethnic minority (Asian, black or other non-white) mothers who were born outside the UK tended to have higher levels of deprivation compared with those born in the UK. ${ }^{16}$ Furthermore, factors associated with deprivation and marital status have been posited to explain about half of the excess PTBs in Afro-Caribbeans. ${ }^{18}$ On the other hand, inconsistent and at times puzzling, associations in the patterns of socioeconomic disadvantage and outcomes in some groups suggest that social disadvantage on its own cannot explain ethnic disparities in infant outcomes in the UK as elsewhere. ${ }^{19} 20$ It has also been postulated that individuals living in 'ethnically dense' areas with members of their own group tend to enjoy better health compared with their counterparts in less ethnically diverse areas. ${ }^{21}$

While individual ethnicity, socioeconomic disadvantage and living in ethnically dense areas (characterised by higher proportions from ethnic minority groups compared with the general population) can all be potentially linked to the risk of preterm birth, studies have seldom explored variations in the risk of PTB among different ethnic groups in settings characterised by high levels of ethnic density and social disadvantage. Focusing on an ethnically dense, socially deprived area in the UK, the objectives of this study were to investigate variations in the risk of PTB, including extreme preterm birth (EPTB) and moderately preterm birth (MPTB), by maternal ethnicity and to examine whether any variations were dependent of area deprivation and maternal biological and behavioural factors.

\section{METHODS}

We conducted a retrospective cross-sectional study using routinely collected anonymous data from the Luton and Dunstable Trust, one of the largest National Health Service maternity units in the UK. The hospital is situated in a socially deprived town, Luton, currently ranked the 59th most deprived area from 326 local authorities in England as defined by the nationally derived Indices of Multiple Deprivation (IMD).$^{22}$ Luton is ethnically dense with approximately $55 \%$ of the population being of Black and Minority Ethnic origin. ${ }^{23}$ The maternity unit delivers approximately 5000 babies per year, providing comprehensive services to all the women in Luton and for significant proportions from a large geographical area in the East of England. We extracted anonymous data from the clinical information system, known as the Ciconia Maternity information System on all women who have had singleton births between April 2007 and March $2016(n=46307)$. Following a preliminary ethical scrutiny of the study by the hospital's research and development office, a separate ethics approval was not deemed to be necessary as the study was based on analysis of routinely collected anonymous data. The hospital's information governance manager ensured adherence to patient confidentiality and data protection before unidentified data were extracted.

\section{Outcome measures}

Gestational age at birth was calculated for each baby by the dating scan if available or from the first day of the mother's last menstrual period to the day of birth and was available for 46305 births. PTB was defined as $<37$ weeks of gestation and further classified into EPTB $(<28$ weeks of gestation) and MPTB (28 to $<37$ weeks of gestation).

\section{Exposure measures}

Self-defined maternal ethnicity was recorded at the mother's first antenatal visit and was available for 45799 (98.9\%) births. Consistent with the 2001 UK census categories, we grouped mothers into white British, white Other (white Irish and any other white background), black Caribbean, black African, Indian, Pakistani, Bangladeshi and Any Other categories.

\section{Covariates}

Covariates were selected a priori based on their associations with the risk of PTB and to ethnic disparities. ${ }^{24}$ The selected covariates were derived based on the information recorded during the first booking appointment and included area-level deprivation (based on mothers' residential postcode), maternal biological factors (age, parity) and prenatal behavioural factors (gestational week at first antenatal appointment, smoking during pregnancy). Maternal age was classified into three categories: ' $\leq 25$ years', '26-35 years' and ' $>35$ years'. Parity was categorised as 'first born', 'second', 'third' and 'fourth or higher order'. Based on the gestational week at first antenatal appointment, mothers were grouped into ' $\leq 12$ weeks' and '>12 weeks'. Using the first four characters of the mothers' residential area postcode, areas were divided into five deprivation quintiles from the least to the most 
deprived based on the nationally derived IMD scores. The IMD measures relative deprivation in small areas or neighbourhoods in England. ${ }^{22}$ The IMD scores are calculated based on 37 separate indicators, organised across seven distinct domains of deprivation including income, employment, health and disability, education, skills and training, crime, barriers to housing and services, and living environment. ${ }^{22}$ The 'first' and the 'fifth' quintiles represented the least and most deprived areas, respectively. There were 45031 births with complete data on gestation, ethnicity and covariates.

\section{Statistical analysis}

We applied logistic regression models to examine the association between ethnicity and PTB. We estimated the ORs and 95\% CIs for preterm (vs full-term) births for each ethnic group, compared with white British. To establish whether the association with ethnicity differed for EPTB and MPTB, we applied multinomial logistic regression models estimating relative risk ratios (RRRs) and 95\% CIs for extreme and moderately preterm (vs full-term) births for each ethnic group, compared with white British. $\mathrm{P}$ values of less than 0.05 were considered as statistically significant. The results were presented using the OR for the estimates from logistic regression and RRR for the estimates from multinomial regression with 95\% CI values.

Both logistic and multinomial models were adjusted in stages. The unadjusted model (model 0 ) included year of birth as a covariate. Subsequent adjustments were made sequentially; first for area-level deprivation (model 1), additionally for maternal biological factors (model 2) and further for prenatal behavioural factors (model 3). Area deprivation was added to the model first as this was a key covariate considered for the study. Prenatal behavioural factors were added in the final model to estimate their additional impact as these factors are potentially amenable to modification with suitable interventions. Main analyses were conducted using a sample of 45031 births with complete data on gestational week, ethnicity and the covariates (97.2\% of all singletons) (online supplementary table S1). All analyses were performed using IBM Statistics Package for the Social Sciences (SPSS) V.21.

\section{Patient and public involvement}

No patients or public were involved in formulating the research question, defining the outcome and exposure measures, analysis and interpretation or writing up of results. No data were directly collected from patients during the course of the study. Where possible, results of the study will be disseminated to the public and patient community and individual patients and families by the authors.

\section{RESULTS}

Of the 46307 singleton births recorded between April 2007 and March 2016, gestational week at birth and ethnicity data were available for 45799 births. There were a total of $3793(8.3 \%)$ PTBs, of which $3236(7.1 \%)$ were
MPTBs and 557 (1.2\%) were EPTBs (table 1). More than one-third $(34.8 \%)$ of the births were to mothers from ethnic minority groups including black Caribbean, black African, Indian, Pakistani and Bangladeshi mothers. Great majority $(85.8 \%)$ of mothers lived in neighbourhoods that were in the three most deprived IMD quintiles, and the proportion was higher for mothers from ethnic minority groups $(97.8 \%)$. Maternal country of birth was recorded for $44.9 \%$ births among which about two-fifths $(42 \%)$ of mothers were born abroad.

\section{Maternal characteristics by ethnic group}

Several characteristics of the sample differed by ethnic group (table 2). Proportions of young mothers (aged $\leq 25$ years) were much lower among Indian $(17.3 \%)$ and black African (17.2\%) mothers than white British mothers $(32.4 \%)$, while the proportions among other ethnic groups ranged from $27.6 \%$ (white Other) to $30.7 \%$ (Bangladeshi). Black Caribbean and black African mothers were more likely to be aged $>35$ years at child birth (20.9\% and $18.9 \%$, respectively), while Bangladeshi $(8.0 \%)$ and Pakistani $(8.8 \%)$ mothers were less likely, compared with white British mothers (13.3\%). More than half of the Indian (51.8\%) and white Other (50.4\%) mothers gave birth to their first baby while these proportions were less among Pakistani (29.3\%) and Bangladeshi $(27.5 \%)$ groups. Smoking during pregnancy was uncommon among ethnic minority mothers in general compared with white British mothers (28.8\%), particularly among Bangladeshi (1.9\%) and Pakistani (2.8\%) mothers. Having the first antenatal appointment after 12 weeks of gestation was most common among black African $(34.2 \%)$, black Caribbean $(29.0 \%)$ and white Other (27.4\%) mothers and was least common among white British (16.4\%) mothers. A great majority of mothers from various ethnic minority groups (86.6\%-98.2\%) lived in the two most deprived IMD quintiles, compared with $47.1 \%$ of white British mothers.

\section{Absolute risk of PTB, EPTB and MPTB among ethnic groups}

The absolute risk (prevalence) of PTB varied across ethnic groups from $7.3 \%$ (95\% CI: $6.7 \%$ to $8.0 \%$ ) for white Other mothers to $9.4 \%$ (8.1\% to $10.9 \%$ ) for Indian mothers. Among ethnic minority groups, Indian mothers had the highest absolute risk of PTB followed by black Caribbean (9.2\% [7.4\% to $11.4 \%]$ ) and black African mothers $(8.8 \%$ [ $7.8 \%$ to $10.0 \%])$, whereas Pakistani (7.5\% [6.9\% to $8.1 \%])$ and Bangladeshi $(7.6 \%$ [6.7\% to $8.6 \%$ ]) mothers had the lowest absolute risk. Overall one-third of PTBs were to mothers from ethnic minority groups (table 1).

The absolute risk of EPTB also varied. Black Caribbean mothers $(2.2 \%$ [ $1.4 \%$ to $3.5 \%])$ had the highest absolute risk of EPTBs, nearly twice as high as white British mothers (1.3\% [1.2\% to $1.5 \%])$. Bangladeshi mothers $(0.6 \%$ [ $0.4 \%$ to $1.0 \%])$ had the absolute lowest risk of EPTBs, nearly half as low as white British mothers. 




Relative risk of PTB, EPTB and MPTB among ethnic groups

The relative risks of PTB, EPTB and MPTB were estimated for the sample of 45031 births with complete data on gestational week, ethnicity and covariates $(97.2 \%$ of all singletons). While black Caribbean ( $\mathrm{OR}=1.09$ [0.86 to 1.38$]$ ) and black African ( $\mathrm{OR}=0.96$ [0.83 to 1.12]) mothers had similar odds of PTB to white British mothers (table 3), they had more relative risk of EPTB, particularly black Caribbean mothers ( $\mathrm{RRR}=1.87$ [1.18 to 2.96]) (table 4). The relative risk of EPTB among black Caribbean mothers exacerbated after adjustment for area deprivation ( $R R R=2.21$ [1.38 to 3.53]) and persisted with marginal reduction after further adjustment for maternal biological and prenatal behavioural factors $(\mathrm{RRR}=1.93$ [1.20 to 3.10]). The relative risk of EPTB among black African mothers compared with white British mothers also exacerbated after adjustment for area deprivation ( RRR=1.63 [1.16 to 2.30]). The differences remained significant, although with reduction after further adjustment for maternal biological factors (RRR=1.57 [1.11 to 2.21]), but became marginal after additional adjustment for prenatal behavioural factors $(\mathrm{RRR}=1.41[0.99$ to 2.01]). The relative risk of MPTB among black Caribbean and black African mothers did not differ from that of white British mothers (table 4).

Pakistani mothers had lower odds of PTB (OR=0.85 [0.77 to 0.96]) compared with white British mothers. The differences became non-significant after adjustment for area deprivation (table 3). There was no significant difference in the relative risk of EPTB between white British and Pakistani mothers. However, Pakistani mothers had lower relative risk of MPTB $(\mathrm{RRR}=0.87$ [0.77 to 0.96$])$; it changed little after adjusting for area deprivation $(\mathrm{RRR}=0.87$ [0.78 to 0.98$])$ and maternal biological factors ( $R R R=0.89$ [0.78 to 0.98$]$ ), but disappeared after adjustment for prenatal behavioural factors ( $R R R=0.96$ [0.85 to 1.08]) (table 4).

The odds of PTB was lower among Bangladeshi mothers $(\mathrm{OR}=0.86[0.74$ to 0.99$])$ (table 3$)$. This was mainly due to a greater reduction in the relative risk of EPTB among Bangladeshi mothers compared with white British mothers $(\mathrm{RRR}=0.48$ [0.30 to 0.78$]$ ); the reduced relative risk remained significant after adjustment for area deprivation ( $R R R=0.59$ [0.36 to 0.96$]$ ), but became non-significant after adjustment for maternal biological factors. The relative risk of MPTB among Bangladeshi mothers did not differ from that of white British mothers (table 4)

The odds of PTB was the lowest among white Other group (OR=0.82 [95\% CI 0.73 to 0.90]) and it changed little after adjustment for covariates $(\mathrm{OR}=0.78$ [95\% CI 0.70 to 0.87 ) (table 3 ). The reduced odds was mainly due to a greater reduction in the relative risk of MPTB among the white Other group compared with the white British group ( $R R R=0.81$ [0.73 to 0.91]) which persisted after adjustment for all covariates ( $R R R=0.79$ [0.70 to 0.89]). There was no significant difference in the relative risk of EPTB between white Other group and the white British group (RRR=0. 83 [0.63 to 1.09 ]) (table 4 ). 





Table 3 OR $(95 \% \mathrm{Cl})$ for preterm birth compared with term birth by ethnic groups $(\mathrm{n}=45031)^{\star}$



Model 0: Unadjusted model: include only ethnic groups and year of birth.

Model 1: Ethnic groups, year of birth and area deprivation.

Model 2: All variables in model 1, plus maternal age and parity.

Model 3: All variables in model 2, plus maternal smoking and gestation at first antenatal visit.

*With complete data on gestation, ethnicity and covariates.

\section{DISCUSSION}

In our cohort of mothers residing in an ethnically dense area with high levels of deprivation, the overall prevalence of PTB $(8.3 \%)$ was higher compared with the national average $(7.8 \%){ }^{2}$ The higher proportions of PTB in this cohort may reflect a high prevalence of risk factors within the pregnant population of the region as a whole. More than two-thirds of the mothers in our cohort lived in areas that were in the two most deprived area quintiles (the most deprived 40\% nationwide). Area deprivation has long been linked to increased risk of $\mathrm{PTB}^{5}$ 7-9 and the overall high PTB prevalence in the cohort could be indicative of this link. Furthermore, some of the prenatal behavioural risk factors such as smoking in pregnancy and delayed initiation of antenatal care were more commonly reported in our cohort compared with mothers nationally. ${ }^{25} 26$

The absolute risk patterns of PTB in terms of prevalence among various ethnic groups in our cohort differed in some respects to the findings from studies using UK nationally representative data. ${ }^{23}$ Nationally, in the UK, black Caribbean, black African, Indian, Bangladeshi and Pakistani, babies have higher prevalence of PTB compared with the white British (7.9\%) with the highest prevalence reported among the black Caribbean group $(10.4 \%) .{ }^{23}$
In our cohort, the differences in the risk of PTB between each ethnic group and the white British was modest with the highest absolute risk reported among Indian mothers followed by black Caribbean mothers. The higher absolute risk of PTB among Indian mothers compared with the white British in our cohort was mainly due to a high risk of MPTB among this group. While nationally, Pakistani babies have been reported to have a slightly higher prevalence of PTB (8\%) compared with white British babies $(7.9 \%),{ }^{2}$ Pakistani mothers in our cohort had the lowest absolute risk among all non-white groups, which was also lower than the white British group, mainly due to a low risk of MPTB among Pakistani mothers. Bangladeshi mothers in our cohort had a lower absolute risk of PTB compared with white British mothers mainly due to a low risk of EPTB which was the lowest among all ethnic groups. White Other group had the lowest absolute risk of PTB among all groups in our cohort consistent with findings from nationally representative data reporting the lowest prevalence rates among this group $(6.6 \%){ }^{2}$ The white British mothers in our cohort had a higher absolute risk of PTB compared with white British babies nationally $(7.9 \%){ }^{2}$

While the national trend showed a threefold prevalence of EPTB among black Caribbean babies compared 


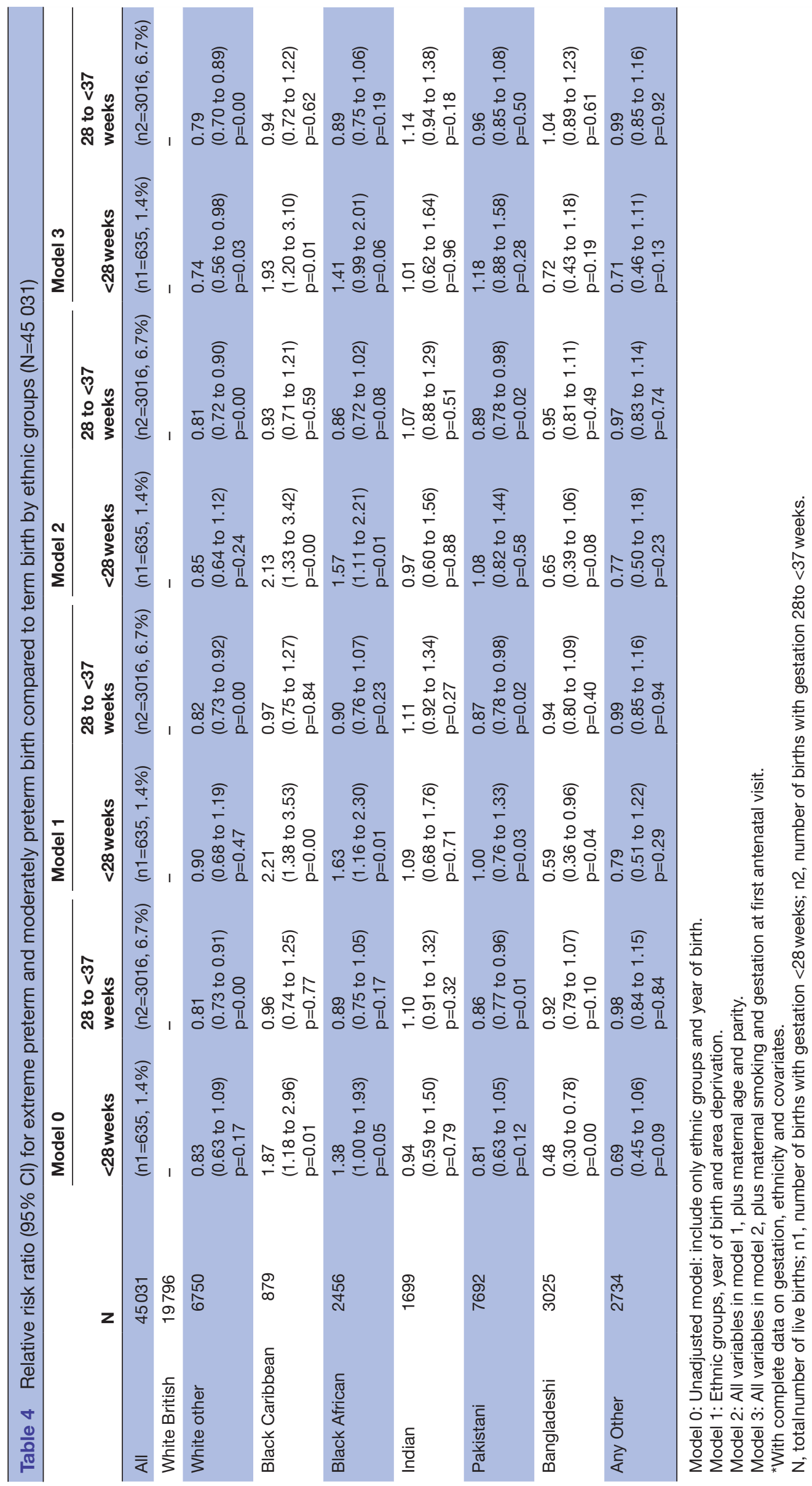


with the white British, ${ }^{23}$ black Caribbean mothers in our cohort had a nearly twofold risk of EPTB compared with white British mothers. Nationally, Pakistani and Bangladeshi mothers tended to have slightly higher prevalence of EPTB $(0.5 \%)^{2}$ compared with white British mothers $(0.4 \%),{ }^{2}$ whereas in our cohort, Pakistani and Bangladeshi mothers had lower risk of EPTB. Overall, many of our findings indicated that there was a reduction in the protective effect found among white British mothers nationally with respect to their risk compared with mothers from ethnic minority groups.

Consistent with findings reported in studies from the $\mathrm{UK},{ }^{27}{ }^{28}$ the proportions of ethnic minority mothers living in the most deprived areas were substantially higher in our cohort-nearly twice-compared with the white British. With respect to maternal biological factors, the percentage of older mothers ( $>35$ years at child birth) were higher among black Caribbean and black African groups compared with the white British group. Timely, adequate, good quality antenatal care is deemed to be an important factor in preventing PTB. ${ }^{29} 30$ Twice the proportions of black African and black Caribbean mothers in our sample initiated antenatal care later than the recommended 12 weeks compared with white British. While smoking is a well-established risk factor for EPTB, substantially higher proportions of white British mothers in our sample smoked at some stage in pregnancy compared with Bangladeshi and Pakistani mothers. Similar findings have been reported from other ethnically diverse socially disadvantaged areas in the UK. ${ }^{31}$

While previous studies have shown a linear relation between deprivation and PTB for all ethnic groups, except for Asians, ${ }^{18}$ our findings indicated that similar levels of area deprivation carry differential implications for some ethnic minority groups in terms of their risk of PTB and EPTB compared with the white British group. For example, the lower odds of PTB disappeared after adjustment for area deprivation among Pakistani and Bangladeshi mothers. For Bangladeshi mothers, the protective effect in terms of their 52\% lower risk of EPTB also diminished after taking area deprivation into account. The increased risk of EPTB among black Caribbean and black African mothers, compared with the white British, increased on an average by $30 \%$ on adjustment to area deprivation. These findings would imply that at similar levels of deprivation, mothers from some ethnic groups were at greater risk compared with white British mothers. However, we found no changes in the odds of PTB for some ethnic groups after adjustment for area deprivation. This may be attributed to small numbers of mothers living in less deprived areas or to the effect of other unadjusted individual socioeconomic confounders. Other plausible explanation could be the link between ethnic density and better health outcomes ${ }^{21}$ which was not directly explored in our study. Previous studies examining associations between ethnic density and PTB have produced inconsistent findings ${ }^{32} 33$ with one study suggesting that high levels of social disadvantage might have inhibited positive effects of ethnic density on health. ${ }^{33}$
The adjustment for maternal biological factors and particularly prenatal behavioural factors among black Caribbean and black African mothers brought some reductions to the increases in relative risk for EPTB that followed adjustment for area deprivation. This would imply that these factors may offset some of the relative risk exacerbated by area deprivation. In the case of black African mothers, the excess risk of EPTB disappeared after adjusting for prenatal behavioural factors implying the role of modifiable factors such as smoking and initiation of antenatal care in explaining the excess risk. Among Pakistani mothers, the protective effect in terms of their $14 \%$ reduced risk of MPTB compared with white British mothers decreased and became non-significant after adjustment for prenatal behavioural factors implying their role in explaining the lower risk. Overall, it would appear that behavioural factors could offer potential explanations to some extent for the higher and lower risk EPTB and MPTB among some ethnic groups compared with the white British.

This is the first study that used a large recent data set to examine ethnic variations in the risk of PTB in an ethnically dense area with high levels of deprivation. Our cohort had substantially higher proportions $(34.8 \%)$ of mothers from ethnic minority groups compared with the maternal population in England and Wales $(12.9 \%)^{2}$ allowing us to explore differences by ethnic group more rigorously. The study has certain limitations, however. Due to the paucity of adequate information, we were unable to examine ethnic variations by country of birth or to include maternal biological factors other than age and parity. The cross-sectional nature of the study may have limited causal inferences. Few ethnic minority mothers in our sample were from less deprived areas and this might have contributed to the fact that ethnic differences in PTB were not always explained by the level of area deprivation. We were unable to account for variations in the length of exposure to the deprived areas as the information was not available in our study. However, residence in deprived areas tends to track overtime for ethnic minority groups, with both old and recent data suggesting that people from ethnic minority groups were more likely than the white British group to live in the most deprived $10 \%$ of neighbourhoods in England, with the exception of the Indian ethnic group. ${ }^{27} 28$ So, this is unlikely to affect our findings. Due to the unavailability of data, we were unable to include other individual-level indicators of socioeconomic status (SES) such as education, employment and household income in the analysis.

\section{CONCLUSION}

The overall high prevalence of PTB found in our ethnically dense maternal cohort, from an area with high levels of deprivation, compared with the national average has implications for antenatal and neonatal care policy and practice nationally and internationally. With increasing global migration, ethnically dense communities in 
pockets of socially deprived neighbourhoods are becoming commonplace in many developed countries, and the mothers in these communities, regardless of their ethnic background, could potentially be at higher risk of PTBs compared with the nation as a whole. The PTB prevalence patterns among various ethnic groups in our cohort reflected differences and consistencies compared with the national trend and overall it would appear that the protective effect found among white British mothers nationally reduced in our sample. Such variations in risk within communities should be taken into consideration while planning services and programmes nationally and locally in settings characterised by high levels of ethnic diversity and disadvantage to ensure equity in provision.

Overall, our findings point to the need to intensify universal and targeted programmes and services to identify and support mothers at risk of PTB in areas with high levels of deprivation. The link between excess risk and modifiable behavioural factors such as smoking during pregnancy and delayed initiation of antenatal care in some ethnic groups points to the need for targeted preventive antenatal interventions. The overall high PTB prevalence has also implications for neonatal care provision in terms of the need for actively incorporating programmes to support parents of preterm babies to minimise associated parental distress and to reduce both short-term and long-term risk of adverse outcomes. The substantially higher and lower risks of EPTB among two ethnic minority groups compared with the white British calls for a detailed exploration of specific protective and risk factors among different ethnic groups in ethnically dense socially disadvantaged neighbourhoods. Future research should include individual indicators of SES in addition to area deprivation to accurately capture the impact of both individual-level and area-level SES on ethnic variations in PTB.

\section{Author affiliations}

${ }^{1}$ Maternal and Child Health Research Centre, Institute for Health Research, University of Bedfordshire, Luton, UK

${ }^{2}$ Population, Policy and Practice Programme, Great Ormond Street Institute of Child Health, University College London, London, UK

${ }^{3}$ Neonatal Unit, Luton and Dunstable University Hospital NHS Foundation Trust, Luton, UK

${ }^{4}$ Independent Consultant, London, UK

${ }^{5}$ Schulich School of Medicine and Dentistry, University of Western Ontario, London, Canada

Acknowledgements The authors would like to thank Sam Okolosi, Gaynor Flynn, Tracey Scivier and Trish Ryan from Luton and Dunstable Hospital Trust for their support in extracting the data; Nathan Shaw and Elliot McBride for their assistance in developing the deprivation quintiles.

Contributors SP took the lead in conceptualising and designing the study, conducted the literature review, coordinated and supervised data extraction and analysis, interpreted the findings and drafted the manuscript. LL assisted with the statistical analysis and interpretation of the data, and critically revised the manuscript for important intellectual content. PcT contributed to the literature review, data analysis and the creation of tables. LK and JK contributed to the conceptualisation and data extraction. TP contributed to the conceptualisation and took the lead in developing the deprivation quintiles and the analysis with respect to area deprivation. AT contributed to the conceptualisation and data interpretation. All authors approved the final manuscript as submitted and agreed to be accountable for all aspects of the work.
Funding The authors have not declared a specific grant for this research from any funding agency in the public, commercial or not-for-profit sectors.

Competing interests None declared.

Patient consent for publication Not required.

Provenance and peer review Not commissioned; externally peer reviewed.

Data sharing statement Due to the nature of the agreement with the data provider, the original data cannot be shared in the public domain.

Open access This is an open access article distributed in accordance with the Creative Commons Attribution Non Commercial (CC BY-NC 4.0) license, which permits others to distribute, remix, adapt, build upon this work non-commercially, and license their derivative works on different terms, provided the original work is properly cited, appropriate credit is given, any changes made indicated, and the use is non-commercial. See: http://creativecommons.org/licenses/by-nc/4.0/.

\section{REFERENCES}

1. World Health Organization. Preterm birth. Fact sheet. 2015 www. who.int/mediacentre/factsheets/fs363/en/ (Accessed 28 May 2017).

2. Office for National Statistics. Birth characteristics in England and Wales: 2016. 2017 https://www.ons.gov.uk/peoplepopulationandc ommunity/birthsdeathsandmarriages/livebirths/bulletins/birthcharact eristicsinenglandandwales/2016 (Accessed 28 May 2017).

3. Office for National Statistics. Childhood mortality in England and Wales: 2015. 2017 https://www.ons.gov.uk/peoplepopulationandc ommunity/birthsdeathsandmarriages/deaths/bulletins/childhoo dinfantandperinatalmortalityinenglandandwales/2015\#immaturityrelated-conditions-remains-most-common-cause-of-infant-deaths (Accessed 28 May 2017).

4. Office for National Statistics. Pregnancy and ethnic factors influencing births and infant mortality: 2013. 2016 https://www. ons.gov.uk/peoplepopulationandcommunity/healthandsocialcare/ causesofdeath/bulletins/pregnancyandethnicfactorsinfluencingbirt hsandinfantmortality/2015-10-14 (Accessed 28 May 2017).

5. Metcalfe A, Lail P, Ghali WA, et al. The association between neighbourhoods and adverse birth outcomes: a systematic review and meta-analysis of multi-level studies. Paediatr Perinat Epidemiol 2011;25:236-45.

6. Goldenberg RL, Culhane JF, lams JD, et al. Epidemiology and causes of preterm birth. Lancet 2008;371:75-84.

7. Bonet M, Smith LK, Pilkington $\mathrm{H}$, et al. Neighbourhood deprivation and very preterm birth in an English and French cohort. BMC Pregnancy Childbirth 2013;13:97.

8. DeFranco EA, Lian M, Muglia LA, et al. Area-level poverty and preterm birth risk: a population-based multilevel analysis. $B M C$ Public Health 2008;8:316.

9. Gray R, Bonellie SR, Chalmers J, et al. Social inequalities in preterm birth in Scotland 1980-2003: findings from an area-based measure of deprivation. BJOG 2008;115:82-90.

10. Weightman AL, Morgan HE, Shepherd MA, et al. Social inequality and infant health in the UK: systematic review and meta-analyses. BMJ Open 2012;2:1-14.

11. Moser K, Stanfield KM, Leon DA. Birthweight and gestational age by ethnic group, England and Wales 2005: introducing new data on births. Health Stat Q 2008;39:34-55.

12. Datta-Nemdharry P, Dattani N, Macfarlane AJ. Birth outcomes for African and Caribbean babies in England and Wales: retrospective analysis of routinely collected data. BMJ Open 2012;2:1-9.

13. Puthussery S. Perinatal outcomes among migrant mothers in the United Kingdom: Is it a matter of biology, behaviour, policy, social determinants or access to health care? Best Pract Res Clin Obstet Gynaecol 2016;32:39-49.

14. Smith LK, Draper ES, Manktelow BN, et al. Socioeconomic inequalities in very preterm birth rates. Arch Dis Child Fetal Neonatal Ed 2007;92:F11-14.

15. Kelly Y, Panico L, Bartley $M$, et al. Why does birthweight vary among ethnic groups in the UK? Findings from the Millennium Cohort Study. $J$ Public Health 2009;31:131-7.

16. Oakley L, Maconochie N, Doyle P, et al. Multivariate analysis of infant death in England and Wales in 2005-06, with focus on socioeconomic status and deprivation. Health Stat Q 2009;42:22-39.

17. Dearden L, Mesnard A, Shaw J. Ethnic differences in birth outcomes in England*. Fisc Stud 2006;27:17-46.

18. Aveyard P, Cheng KK, Manaseki S, et al. The risk of preterm delivery in women from different ethnic groups. BJOG 2002;109:894-9.

19. Gray R, Headley J, Oakley L, et al. Inequalities in infant mortality project briefing paper 3 . Towards an understanding of variations in infant mortality rates between different ethnic groups. 2009. 
20. Teitler JO, Reichman NE, Nepomnyaschy L, et al. A cross-national comparison of racial and ethnic disparities in low birth weight in the United States and England. Pediatrics 2007;120:e1182-9.

21. Whitley R, Prince M, McKenzie K, et al. Exploring the ethnic density effect: a qualitative study of a London electoral ward. Int J Soc Psychiatry 2006;52:376-91.

22. UK Department for Communities and Local Government. The English indices of deprivation 2015. 2015 https://www.gov.uk/government/ uploads/system/uploads/attachment_data/file/465791/English_ Indices_of_Deprivation_2015_-_Statistical_Release.pdf (Accessed 01 Jul 2017).

23. Luton Borough Council. Healthy people. 2013 https://www.luton.gov. uk/Community_and_living/Lists/LutonDocuments/PDF/JSNA/2\% 20Healthy\%20People.pdf (Accessed 01 Jul 2017).

24. Alexander GR, Slay M. Prematurity at birth: trends, racial disparities, and epidemiology. Ment Retard Dev Disabil Res Rev 2002;8:215-20.

25. National Health Service Digital. Statistics on women's smoking status at time of delivery. $2017 \mathrm{https}$ ///files.digital.nhs.uk/publication/6/r/ stat-wome-smok-time-deli-eng-q4-16-17-rep.pdf (Accessed 10 Dec 2018).

26. National Health Service Digital. Maternity Services Monthly Statistics England - January 2018. $2018 \mathrm{https} / / /$ digital.nhs.uk/data-andinformation/publications/statistical/maternity-services-monthlystatistics/january-2018 (Accessed Jul 2018).
27. Barnard H, Turner C. Poverty and ethnicity: a review of evidence. https://www.jrf.org.uk/sites/default/files/jrf/migrated/files/povertyethnicity-evidence-summary.pdf (Accessed 01 Sep 2018).

28. UK Department for Communities and Local Government. People living in deprived neighbourhoods. $2018 \mathrm{https} / / / \mathrm{www}$.ethnicityfacts-figures.service.gov.uk/british-population/demographics/ people-living-in-deprived-neighbourhoods/latest (Accessed $01 \mathrm{Sep}$ 2018).

29. Cox RG, Zhang L, Zotti ME, et al. Prenatal care utilization in Mississippi: racial disparities and implications for unfavorable birth outcomes. Matern Child Health J 2011;15:931-42.

30. Wehby GL, Pawluk M, Nyarko KA, et al. Explaining ethnic disparities in preterm birth in Argentina and Ecuador. Glob Public Health 2018;13:1126-43.

31. Stacey T, Prady S, Haith-Cooper M, et al. Ethno-specific risk factors for adverse pregnancy outcomes: findings from the born in Bradford Cohort Study. Matern Child Health J 2016;20:1394-404.

32. Pickett KE, Shaw RJ, Atkin K, et al. Ethnic density effects on maternal and infant health in the Millennium Cohort Study. Soc Sci Med 2009;69:1476-83.

33. Uphoff EP, Pickett KE, Crouch S, et al. Is ethnic density associated with health in a context of social disadvantage? Findings from the Born in Bradford cohort. Ethn Health 2016;21:196-213. 